\title{
Synaptic protein UNC-13 interacts with an F-box protein that may target it for degradation by proteasomes $\star$
}

\author{
Cristina Polinsky, Chanelle Houston, Jaynine Vado, Azizahmed Shaikh \\ and Rebecca E. Kohn ${ }^{凶}$ \\ Department of Biology, Ursinus College, Collegeville, PA, USA; ${ }_{e}$-mail: rkohn@ursinus.edu
}

Received: 26 May, 2005; revised: 18 November, 2005; accepted: 19 November, 2005

available on-line: 23 February, 2006

\begin{abstract}
UNC-13 protein participates in regulating neurotransmitter release. In Drosophila melanogaster, proteasomal degradation controls UNC-13 levels at synapses. Function of the amino-terminal region of a $207 \mathrm{kDa}$ form of Caenorhabditis elegans UNC-13 is unknown. Yeast two-hybrid and secondary yeast assays identified an F-box protein that interacts with this amino-terminal region. As F-box proteins bind proteins targeted for proteasomal degradation, this protein may participate in degrading a subset of UNC-13 proteins, suggesting that different forms of UNC-13 are regulated differently. Yeast assays also identified an exonuclease, a predicted splicing factor, and a protein with coiled-coil domains, indicating that UNC-13 may affect RNA function.
\end{abstract}

Keywords: synaptic neurotransmission, yeast two-hybrid, UNC-13, F-box

Release of neurotransmitters into synapses requires synaptic vesicle docking, priming and fusion with the neuronal plasma membrane. Proteins on the synaptic vesicles and proteins associated with the plasma membrane form a soluble $N$-ethylmaleimide-sensitive fusion protein attachment protein receptor (SNARE) complex that holds synaptic vesicles adjacent to the plasma membrane. Following maturation into a fusion-competent state, fusion of vesicles with the plasma membrane is triggered by the presence of calcium. UNC-13 proteins interacts with several proteins involved in regulating neurotransmitter release including Doc2, $\beta$-spectrin, RIM-1, UNC-18, and syntaxin (Betz et al., 1997; 2001; Orita et al., 1997; Sakaguchi et al., 1998; Sassa et al., 1999). UNC-13 promotes a change in syntaxin from a closed to open conformation so that it can associate with synaptobrevin and SNAP-25 in the SNARE complex (Richmond et al., 2001). UNC-13 function is regulated by signal transduction pathways. In Dro-

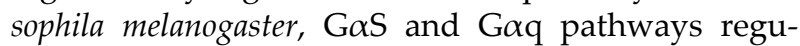
late abundance of Drosophila UNC-13 (DUNC-13) at synapses through proteasomal degradation (Arava- mudan \& Broadie, 2003). In Caenorhabditis elegans, $\mathrm{G} \alpha \mathrm{o}$ and $\mathrm{G} \alpha \mathrm{q}$ pathways regulate localization of a $167 \mathrm{kDa}$ form of UNC-13 to synapses (Lackner et al., 1999; Miller et al., 1999; Nurrish et al., 1999).

In C. elegans, expression of two different unc13 transcripts has been verified with Northern transfers. The more abundant transcript is $5.4 \mathrm{~kb}$ and codes for a $207 \mathrm{kDa}$ protein and the less abundant transcript is $4.4 \mathrm{~kb}$ and codes for a $167 \mathrm{kDa}$ protein. The $5^{\prime}$ region of the two transcripts varies. There are 13 exons at the $5^{\prime}$ end of the $5.4 \mathrm{~kb}$ transcript that are absent in the $4.4 \mathrm{~kb}$ transcript and one exon at the $5^{\prime}$ end of the $4.4 \mathrm{~kb}$ transcript that is absent in the $5.4 \mathrm{~kb}$ transcript. The $3^{\prime}$ region of both transcripts has 16 identical exons (Kohn et al., 2000). The carboxyl-terminal region of the protein that is coded for by both transcripts interacts with syntaxin (Betz et al., 1997). Functions of the dissimilar amino-terminal regions of the two UNC-13 forms have not been characterized. Antibodies recognizing the amino-terminal region of the $207 \mathrm{kDa}$ protein label synapses in the nervous system and nuclei in the gut and gonad (Kohn et al., 2000). Green fluorescent protein

$\star$ Preliminary report was presented at the 15th Biennial C. elegans Conference, University of California, 25-29 June 2005, Los Angeles, California, USA.

Abbreviations: 3AT, 3-aminotriazole; DUNC-13, Drosophila melanogaster UNC-13; SNARE, soluble N-ethylmaleimide-sensitive fusion protein attachment protein receptor; $\mathrm{SD}$, synthetic dextrose. 
fusion analysis of $167 \mathrm{kDa} \mathrm{UNC}-13$ indicates that it is found along axons and that $\mathrm{G} \alpha \mathrm{o}$ and $\mathrm{G} \alpha \mathrm{q}$ signal transduction pathways regulate localization of the protein to synapses (Lackner et al., 1999; Miller et al., 1999; Nurrish et al., 1999).

To further understand function of UNC-13 we investigated the role of the amino-terminal region of the $207 \mathrm{kDa}$ form of UNC-13 expressed in C. elegans. We identified interacting proteins using a yeast two-hybrid screen. The strength of the proteinprotein interactions was examined using three secondary yeast screens. Four proteins that have strong protein-protein interactions with the amino-terminal region of $207 \mathrm{kDa}$ UNC-13 were identified. One is an F-box protein that may regulate levels of $207 \mathrm{kDa}$ UNC-13 at synapses via proteasomal degradation. The other three are ERI-1, which regulates RNA interference, a protein predicted to be a splicing factor, and a protein with two coiled-coil domains. Interaction with these three proteins suggests that in addition to playing a role in regulating neurotransmitter release, UNC-13 may be involved in regulating the processing and function of RNA.

\section{MATERIALS AND METHODS}

Plasmids for yeast two-hybrid screen. cDNA corresponding to unc-13 exons 1-13 was made using Super Script ${ }^{\mathrm{TM}}$ II Reverse Transcriptase (Invitrogen $^{\mathrm{TM}}$ Life Technologies, Carlsbad, California, USA) according to manufacturer's directions. Reverse primer oRK107 5'CTT AAT TCC GAG ATC AGC ACA 3' was used to generate the CDNA and forward primer oRK105 5'CACC ATG GAT GAC GTT GGA GAT3' was used in the subsequent PCR reaction. unc-13 cDNA was transferred into a plasmid using the pENTR $^{\mathrm{TM}}$ Directional TOPO Cloning Kit (Invitrogen $^{\mathrm{TM}}$ Life Technologies, Carlsbad, California, USA) according to manufacturer's directions. Following sequence verification, the unc- 13 cDNA was transferred into the pDEST32 vector coding for the Gal4 DNA binding domain using the ProQuest ${ }^{\mathrm{TM}}$ Two-Hybrid System with Gateway ${ }^{\circledR}$ Technology (Invitrogen $^{\mathrm{TM}}$ Life Technologies, Carlsbad, California, USA) according to manufacturer's directions. A positive clone of unc-13 cDNA corresponding to exons 1-13 in the pDEST32 vector (pDB-UNC13N) was purified using a QIAprep Spin Miniprep Kit (Qiagen, Valencia, California, USA), according to manufacturer's instructions, and verified by sequencing at the Children's Hospital of Philadelphia (Philadelphia, PA, USA).

Yeast two-hybrid screen. The screen was carried out using bait and prey vectors, yeast strain, and controls included in the ProQuest ${ }^{\mathrm{TM}}$ Two-Hybrid System with Gateway ${ }^{\circledR}$ Technology (Invitro- gen $^{\mathrm{TM}}$ Life Technologies, Carlsbad, California, USA). Empty bait vector pDBLeu and UNC-13 bait vector pDB-UNC13N were transformed into yeast strain MaV203 and plated on minimal media lacking leucine (synthetic dextrose (SD)-Leu). Empty activation vector pEXP-AD502 was transformed into yeast strain MaV203 with pDBLeu or pDB-UNC13N and plated on minimal media lacking leucine and tryptophan (SD-Leu-Trp). Controls A through D correspond to different levels of protein-protein interactions with control A having no interaction, control $\mathrm{B}$ having a weak interaction, control $\mathrm{C}$ having a moderately strong interaction and control D having a strong interaction. 3-Aminotriazole (3AT) concentration needed to titrate basal levels of HIS3 expression was determined by plating MaV302 cells with pDBLeu and pEXP-AD502, MaV302 cells with pDB-UNC13N and pEXP-AD502, and controls A-D on SD-Leu-Trp-His plates containing concentrations of $3 \mathrm{AT}$ at $10 \mathrm{mM}, 25 \mathrm{mM}, 50 \mathrm{mM}, 75 \mathrm{mM}$, and $100 \mathrm{mM}$. All plates lacking histidine in the screen had $50 \mathrm{mM}$ 3AT added. MaV203 cells with pDBUNC13N were transformed with cDNA library (Li et al., 2004) and were plated on SD-Leu-Trp and SDLeu-Trp-His $+50 \mathrm{mM}$ 3AT plates. Thirteen C. elegans genomes were screened.

Secondary yeast screens. Secondary screens were carried out according to ProQuest ${ }^{\mathrm{TM}}$ TwoHybrid System with Gateway ${ }^{\circledR}$ Technology (InvitrogenTM Life Technologies, Carlsbad, California, USA) protocols. Colonies that grew on the SD-LeuTrp-His $+50 \mathrm{mM}$ 3AT plates following transformation with a cDNA library and controls A-D were streaked on SD-Leu-Trp and SD-Leu-Trp-His+50 mM 3AT plates. Yeast on the SD-Leu-Trp plate were replica plated onto a SD-Leu-Trp, a YPD plate with a nitrocellulose filter on top, SD-Leu-Trp-His $+50 \mathrm{mM}$ 3AT plate, SD-Leu-Trp-Ura, and SD-Leu-Trp-His +50 $\mathrm{mM} 3 \mathrm{AT}+$ cycloheximide. The SD-Leu-Trp and YPD + nitrocellulose filter plates were incubated i mMediately and remaining plates were replica cleaned and then incubated. The nitrocellulose filter was assayed for $\beta$-galactosidase expression according to manufacturer's instructions. Four plasmids from yeast expressing all three reporter genes and sensitivity to cycloheximide were purified using a QIAprep Spin Miniprep Kit (Qiagen, Valencia, Ca, USA), according to manufacturer's instructions, and verified by sequencing at the Children's Hospital of Philadelphia (Philadelphia, PA, USA).

\section{RESULTS AND DISCUSSION}

In a yeast two-hybrid screen for proteins interacting with the amino-terminal region of $C$. elegans $207 \mathrm{kDa} \mathrm{UNC}-13$, seven candidates were 
identified. Candidates were tested with four secondary yeast screens to examine the strength of the protein-protein interactions. Four candidates grew in the absence of histidine and in the absence of uracil following replica plating and replica cleaning (Fig. 1). These candidates therefore express both the HIS3 and URA3 reporter genes as a result of protein-protein interactions with the amino-terminal region of $207 \mathrm{kDa}$ UNC-13. All four candidates grew in a similar manner as control $\mathrm{D}$, indicating a strong protein-protein interaction. None of the candidates grew in the presence of cycloheximide following replica plating and replica cleaning (Fig. 1). Growth on cycloheximide would have indicated that the yeast cells lost the pDB-UNC13N bait plasmid that expresses the dominant gene $\mathrm{C} Y H 2^{S}$ and sensitizes cells to cycloheximide. In a $\beta$-galactosidase assay, the same four candidates that grew in the absence of histidine and uracil turned dark blue and
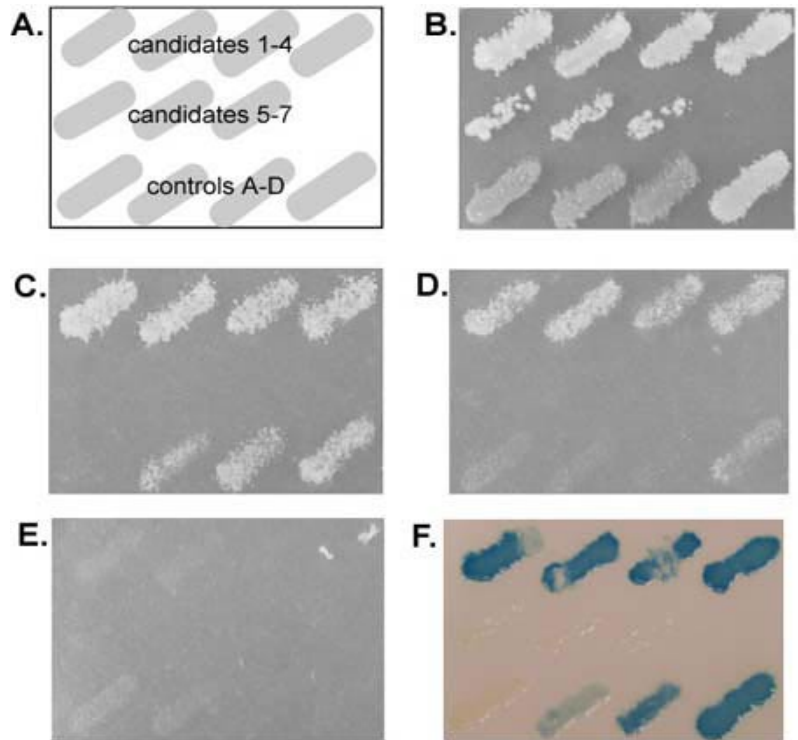

Figure 1. Secondary yeast screens analyzing strength of protein-protein interactions.

(A) Identities of yeast patches are described. The top row in each panel includes candidates one through four. The second row includes candidates five through seven. The bottom row includes controls A through D. (B) All candidates for protein-protein interactions and all controls are shown growing on plate lacking leucine and tryptophan. (C) Candidates one through four and controls B, C, and D are shown growing on a plate lacking leucine, tryptophan, and histidine, with $50 \mathrm{mM}$ 3AT. Candidates five through seven and control A show no growth. (D) Candidates one through four and control D are shown growing on plates lacking leucine, tryptophan, and uracil. Candidates five through seven show no growth and controls A, B, and $C$ show little growth. (E) In the presence of cycloheximide candidates three through seven and controls $C$ and D show no growth. Slight growth is seen for candidates one and two and controls A and B. (F) $\beta$-Galactosidase expression assay shows candidates one through four as dark blue. Candidates five through seven did not turn blue. Control A is not blue and controls B, C, and D have increasing intensity of blue color. were the same intensity as control $\mathrm{D}$, indicating a strong protein-protein interaction. The remaining three candidates were the same color as control A, indicating no protein-protein interaction (Fig. 1). The four cDNAs coding for proteins that interact with the amino-terminal region of $207 \mathrm{kDa}$ UNC-13 were sequenced and identified as 1) $C$. elegans gene coding for a protein with F-box and FTH domains (GenBank accession number NM_074480); 2) C. elegans gene eri-1 (GenBank accession number NM_ 171245), coding for a protein with exonuclease and SAP domains that is involved in regulating RNA interference; 3) C. elegans gene coding for an RNA recognition motif and PWI domain that is predicted to be a splicing factor (GenBank accession number NM_073733); and 4) C. elegans gene coding for a protein with two coiled-coil domains (GenBank accession number NM_67630).

The F-box protein identified in this screen could play a role in regulating levels of UNC-13 available at synapses. DUNC-13 is degraded in Drosophila melanogaster by proteasomes responding to $\mathrm{G} \alpha \mathrm{S}$ and Gaq pathways (Aravamudan \& Broadie, 2003). F-box proteins are components of ubiquitin ligase complexes (Skowyra et al., 1997). The F-box protein binds to a target protein that is then ubiquitinated. Following ubiquitination, the target protein is degraded by proteasomes (Hershko \& Ciechanover, 1998). There are 326 predicted F-box proteins in C. elegans, most of which have unknown functions (Kipreos \& Pagano, 2000). As the F-box protein identified in this study interacts with the amino-terminal end of the $207 \mathrm{kDa}$ UNC-13 product, an amino-acid sequence absent from the $167 \mathrm{kDa}$ product, it would potentially target only a subset of UNC-13 proteins for degradation. There are 20 lysine residues in $207 \mathrm{kDa}$ UNC-13 that could be targets for ubiquitination (Pickart, 2001). Ninety eight additional lysine residues are found in the 1206 amino acids in co mMon between the $207 \mathrm{kDa}$ and $167 \mathrm{kDa}$ forms of UNC-13. A mechanism for degrading a subset of UNC-13 proteins would allow levels of the $207 \mathrm{kDa}$ and $167 \mathrm{kDa}$ protein products expressed from the unc-13 gene to be differentially regulated, suggesting that the two protein products have different functions.

Three additional proteins that interact with the amino-terminal end of $207 \mathrm{kDa}$ UNC-13 were identified in this screen and implicate $u n c-13$ in roles beyond regulating neurotransmitter release. The eri-1 gene codes for an exonuclease that negatively regulates RNA interference. The protein is expressed in the cytoplasm of a subset of neurons and in the gonad. Few mRNAs in the C. elegans nervous system are degraded as a result of RNA interference and it has been suggested that ERI-1 may be involved in limiting cell-type specificity of RNA interference (Kennedy et al., 2004). A protein with RNA recog- 
nition and PWI domains that it is predicted to be a splicing factor and a protein with two coiled-coil domains were also identified as interacting with 207 $\mathrm{kDa}$ UNC-13. A previous yeast two-hybrid screen indicated that the protein with two coiled-coil domains also interacts with the product of the $h p l-2$ gene in C. elegans ( $\mathrm{Li}$ et al., 2004). hpl-2 is involved in formation of the germline, silencing of transgenes in the germline, and in vulval development (Couteau et al., 2002). Interactions between UNC-13 and proteins that function in the nervous system, nuclei, and germline convey the significance of antibodies specific for the amino-terminal end of $207 \mathrm{kDa}$ UNC-13 labeling synapses as well as nuclei in the gut and gonad (Kohn et al., 2000). Direct proteinprotein interactions between UNC-13 and ERI-1 may include UNC-13 in a mechanism regulating RNA interference in the nervous system. Interactions with a splicing factor indicate a possible role for $207 \mathrm{kDa}$ UNC-13 in regulating processing of RNA in nuclei of the gut and gonad. Association with a coiled-coil domain protein that interacts with HPL-2 suggests that UNC-13 protein in gonad nuclei could participate in cell-type specific silencing of transgenes and formation of the germline.

\section{Acknowledgements}

We thank Philip Meneely, Nate Favini, and Lauren Ortiz for their help and support with the yeast two-hybrid screens. We also thank Philip Meneely for providing high quality C. elegans RNA for cDNA production. We thank Theresa Moser for her technical support in the laboratory and Rebecca Roberts for critical reading of the manuscript.

This material is based upon work supported by the National Science Foundation under Grant No. 0092733 to R.E.K.

\section{REFERENCES}

Aravamudan B, Broadie K (2003) Synaptic Drosophila UNC13 is regulated by antagonistic G-protein pathways via a proteasome-dependent degradation mechanism. J Neurobiol 54: 417-438.

Betz A, Okamoto M, Benseler F, Brose N (1997) Direct interaction of the rat unc-13 homologue Munc13-1 with the $\mathrm{N}$ terminus of syntaxin. J Biol Chem 272: 2520-2526.

Betz A, Thakur P, Junge HJ, Ashery U, Rhee J, Scheuss V, Rosenmund C, Rettig J, Brose N (2001) Functional interaction of the active zone proteins munc13-1 and rim1 in synaptic vesicle priming. Neuron 30: 183-196.
Couteau F, Guerry F, Muller F, Palladino F (2002) A heterochromatin protein 1 homologue in Caenorhabditis elegans acts in germline and vulval development. EMBO Rep 3: 235-241.

Hershko A, Ciechanover A (1998) The ubiquitin system. Annu Rev Biochem 67: 425-479.

Kennedy S, Wang D, Ruvkun G (2004) A conserved siRNA-degrading RNase negatively regulates RNA interference in C. elegans. Nature 427: 645-649.

Kipreos ET, Pagano M (2000) The F-box protein family. Genome Biol 1: reviews 3002.1-3002.7.

Kohn RE, Duerr JS, McManus JR, Duke A, Rakow TL, Maruyama H, Moulder G, Maruyama IN, Barstead RJ, Rand JB (2000) Expression of multiple UNC-13 proteins in the Caenorhabditis elegans nervous system. Mol Biol Cell 11: 3441-3452.

Lackner MR, Nurrish SJ, Kaplan JM (1999) Facilitation of synaptic transmission by EGL-30 Gqalpha and EGL-8 PLCbeta: DAG binding to UNC-13 is required to stimulate acetylcholine release. Neuron 24: 335-346.

Li S, Armstrong CM, Bertin N, Ge H, Milstein S, Boxem M, Vidalain PO, Han JD, Chesneau A, Hao T, Goldberg DS, Li N, Martinez M, Rual JF, Lamesch P, Xu L, Tewari M, Wong SL, Zhang LV, Berriz GF, Jacotot L, Vaglio P, Reboul J, Hirozane-Kishikawa T, Li Q, Gabel HW, Elewa A, Baumgartner B, Rose DJ, Yu H, Bosak S, Sequerra R, Fraser A, Mango SE, Saxton WM, Strome S, Van Den Heuvel S, Piano F, Vandenhaute J, Sardet C, Gerstein M, Doucette-Sta mM L, Gunsalus KC, Harper JW, Cusick ME, Roth FP, Hill DE, Vidal M (2004) A map of the interactome network of the metazoan C. elegans. Science 303: 540-543.

Miller KG, Emerson MD, Rand JB (1999) Galpha and diacylglycerol kinase negatively regulate the $\mathrm{G}_{\mathrm{q}}$ alpha pathway in C. elegans. Neuron 24: 323-333.

Nurrish S, Segalat L, Kaplan JM (1999) Serotonin inhibition of synaptic transmission: Galpha(0) decreases the abundance of UNC-13 at release sites. Neuron 24: 231-242.

Orita S, Naito A, Sakaguchi G, Maeda M, Igarashi H, Sasaki T, Takai Y (1997) Physical and functional interactions of Doc2 and Munc13 in $\mathrm{Ca}^{2+}$ dependent exocytotic machinery. J Biol Chem 272: 16081-16084.

Pickart CM (2001) Mechanisms underlying ubiquitination. Annu Rev Biochem 70: 503-533.

Richmond JE, Weimer RM, Jorgensen EM (2001) An open form of syntaxin bypasses the requirement for UNC-13 in vesicle priming. Nature 412: 338-341.

Sakaguchi G, Orita S, Naito A, Maeda M, Igarashi H, Sasaki T, Takai Y (1998) A novel brain-specific isoform of beta spectrin: isolation and its interaction with Munc13. Biochem Biophys Res Co mMun 248: 846-851.

Sassa T, Harada S, Ogawa H, Rand JB, Maruyama IN, Hosono R (1999) Regulation of the UNC-18-Caenorhabditis elegans syntaxin complex by UNC-13. J Neurosci 19: 4772-4777.

Skowyra D, Craig KL, Tyers M, Elledge SJ, Harper JW (1997) F-Box proteins are receptors that recruit phosphorylated substrates to the SCF ubiquitin-ligase complex. Cell 91: 209-219. 